\section{Intestinal tuft cell function}

Tuft cells have long been a mysterious component of the intestinal epithelium. Related papers by two groups in Nature and one group in Science demonstrate important roles for tuft cells in sensing and responding to intestinal protozoa and helminth parasites. Tuft cells normally make up a small fraction of the intestinal epithelium, but after exposure to parasites, they substantially increase in number and release copious amounts of interleukin 25 (IL-25). That IL-25, in turn, activates group 2 innate lymphoid cells, which then drive a type 2 immune response characterized by IL-13, eosinophila and mucus production; these collectively lead to expulsion of the parasite. Deletion of tuft cells or ablation of their chemosensory function by knockout of the cation channel TRPM5 impairs the type 2 response and parasite expulsion. This demonstration that tuft cells are prominent IL-25 producers indicates a key role for these cells in the initiation of type 2 immune responses in the small intestine. Nature 529, 221-225 \& 226-230 (2016); Science (4 February 2016) doi: $10.1126 /$ science.aaf 1648

\section{HIV-1 trans-infection}

Although human dendritic cells (DCs) are largely resistant to productive infection with human immunodeficiency virus type 1 (HIV-1), they efficiently transmit the virus to $\mathrm{T}$ cells through a process called 'trans-infection'. In Cell, Ménager and Littman screen human monocyte-derived DCs with short hairpin RNA and identify the membrane protein TSPAN7 and the actin nucleator DNM2 as regulators of T cell trans-infection. TSPN7-deficient DCs show loss of dendrites, while both DNM2- as well as TSPN7-deficient DCs show redistribution of HIV-1 particles to the cytoplasmic compartment, increased HIV-1 localization in micropinosomes and reduced trans-infection. Inhibition of endocytosis and macropinocytosis or restoration of HIV-1 localization on dendrites enhances trans-infection. These results indicate that the internalization of HIV-1 by DCs through various mechanisms diminishes its transfer to T cells.

Cell 164, 695-709 (2016)

\section{Exercising NK cells}

Regular exercise confers many health benefits. In Cell Metabolism, Pedersen et al. show that the benefits of exercise include enthanced anti-tumor resistance. Mice with free access to running wheels have diminished tumor incidence and burden in multiple tumor models. Exercise triggers the release of epinephrine, which enhances the mobilization and tumor infiltration of NK cells. NK cells also recruit other lymphocytes to the tumors; however, only ablation of NK cell populations abolishes the anti-tumor effects. Exercise also increases the systemic release of IL-6, which contributes to the anti-tumor response. Injection of epinephrine, but not of IL- 6 alone, mimics the anti-tumor effects of exercise. These findings show NK cells are the dominant population that is responsive to exerciseinduced anti-tumor responses.

$L A D$ Cell Metab. (8 March 2016) doi:10.1016/j.cmet.2016.01.011

\section{Lymphocytes in Alzheimer's disease}

Components of the innate immune system such as microglia have been well studied in Alzheimer's disease (AD), but the role of lymphocytes and cells of the adaptive immune system is less well understood. In the Proceedings of the National Academy of Sciences, Blurton-Jones and colleagues develop an AD-prone mouse model deficient in lymphocytes ( $\mathrm{T}$ cells, B cells and natural killer (NK) cells) to determine the role of the adaptive immune system in the progression of $\mathrm{AD}$. These 'Rag-5xfAD' mice show greater accumulation of amyloid $A \beta$ plaques in the brain and enhanced signs of microglia activation-both signatures of $\mathrm{AD}$ progression. The accumulation of $\mathrm{A} \beta$ in Rag-5xfAD mice seems to be due to an impairment in $A \beta$ phagocytosis rather than greater $A \beta$ production. Nonspecific immunoglobulins associated with microglia in lymphoreplete mice or in Rag-5xfAD that received bulk immunoglobulins seem to trigger $A \beta$ phagocytosis and clearance. These data suggest that lymphocytes can slow AD by 'dialog' with microglia and thereby facilitate $\mathrm{A} \beta$ clearance.

Proc. Natl. Acad. Sci. USA (16 February 2016) doi:10.1073/ pnas. 1525466113

\section{The scorpion sting}

Scorpion venom induces massive release of pro-inflammatory cytokines, such as IL-1 $\beta$, IL- 6 and TNF, and multi-organ failure, including pulmonary edema. In Nature Communications, Zoccal et al. show that in mice, Tityus serrulatus venom induces activation of the NLRP3 inflammasome and that IL- $1 \beta$ production is regulated by the balance between the lipid mediators $\mathrm{PGE}_{2}$ and $\mathrm{LTB}_{4}$. Signaling via the receptor for IL-1 is required for inflammation and neutrophil accumulation in the lungs, as well as for the secretion of $\mathrm{LTB}_{4}$ and $\mathrm{PGE}_{2}$. Alox5 $5^{-1-}$ mice, which have defective $\mathrm{LTB}_{4}$ production, show greater venom-induced IL-1 $\beta$, inflammation and mortality than that of wild-type mice, which indicates that 5-lipoxygenase metabolites such as LTB $_{4}$ are protective against excessive inflammation. In contrast, $\mathrm{PGE}_{2}$ induces IL-1 $\beta$ though a pathway involving CAMP, the kinase PKA and the transcription factor NF- $\mathrm{kB}$, and inhibition of $\mathrm{PGE}_{2}$ by the targeting of COX cyclooxygenases is protective against inflammation and lethality. This suggests that COX inhibitors such as indomethacin could be used therapeutically against scorpion stings. Nat. Commun. (23 February 2016) doi:10.1038/ncomms 10760

\section{Fighting bacteria}

The administration of antibiotics causes the dysbiosis of commensal microbial populations, which can lead to colonization by vancomycinresistant Enterococcus faecium (VRE) and, potentially, sepsis in compromised patients. In Science Translational Medicine, Pamer and colleagues show that triggering antiviral innate immunity restores resistance to VRE. The receptor TLR7 normally recognizes viral RNA but can be stimulated with the synthetic ligand resiquimod (R-848). Oral administration of R-848 stimulates CD11c ${ }^{+}$dendritic cells to induce expression of IL-23. In turn, IL-23 triggers IL-22 expression in gut innate lymphoid cells, which leads to the expression of antimicrobial peptides, including Reg $3 \gamma$. This innate immune response helps to diminish VRE numbers in the gut after antibiotic-induced dysbiosis, which suggests a means for helping to control enterococcal infections in vunerable patients.

$L A D$ 